\title{
Probing the Metal Enrichment of the Intergalactic Medium at $z=5-6$ Using the Hubble Space Telescope
}

\author{
Zheng Cai ${ }^{1,8}$ (1) Xiaohui Fan ${ }^{2}$ (1), Romeel Dave ${ }^{3,4,5}$ (D) Kristian Finlator $^{6}$, and Ben Oppenheimer ${ }^{7}$ (1) \\ ${ }^{1}$ UCO/Lick Observatory, University of California, 1156 High Street, Santa Cruz, CA 95064, USA; zcai@ucolick.org \\ 2 Steward Observatory, University of Arizona, 933 North Cherry Avenue, Tucson, AZ 85721, USA \\ ${ }^{3}$ Institute for Astronomy, University of Edinburgh, Royal Observatory, Edinburgh EH9 3HJ, UK \\ ${ }^{4}$ University of the Western Cape, Bellville, Cape Town 7535, South Africa \\ ${ }^{5}$ South African Astronomical Observatories, Observatory, Cape Town 7925, South Africa \\ ${ }^{6}$ New Mexico State University, Las Cruces, NM 88003, USA \\ ${ }^{7}$ CASA, Department of Astrophysical and Planetary Sciences, University of Colorado, 389 UCB, Boulder, CO 80309, USA \\ Received 2017 July 7; revised 2017 September 27; accepted 2017 September 27; published 2017 October 26
}

\begin{abstract}
We test the galactic outflow model by probing associated galaxies of four strong intergalactic C IV absorbers at $z=5-6$ using the Hubble Space Telescope (HST) Advanced Camera for Surveys (ACS) ramp narrowband filters. The four strong C IV absorbers reside at $z=5.74,5.52,4.95$, and 4.87, with column densities ranging from $N_{\text {C IV }}=10^{13.8}$ to $10^{14.8} \mathrm{~cm}^{-2}$. At $z=5.74$, we detect an i-dropout Ly $\alpha$ emitter (LAE) candidate with a projected impact parameter of 42 physical kpc from the C IV absorber. This LAE candidate has a Ly $\alpha$-based star formation rate $\left(\mathrm{SFR}_{\mathrm{Ly} \alpha}\right)$ of $2 M_{\odot} \mathrm{yr}^{-1}$ and a UV-based SFR of $4 M_{\odot} \mathrm{yr}^{-1}$. Although we cannot completely rule out that this i-dropout emitter may be an [O II] interloper, its measured properties are consistent with the C IV powered galaxy at $z=5.74$. For C IV absorbers at $z=4.95$ and $z=4.87$, although we detect two LAE candidates with impact parameters of 160 and $200 \mathrm{kpc}$, such distances are larger than that predicted from the simulations. Therefore, we treat them as nondetections. For the system at $z=5.52$, we do not detect LAE candidates, placing a $3 \sigma$ upper limit of $\mathrm{SFR}_{\mathrm{Ly} \alpha} \approx 1.5 M_{\odot} \mathrm{yr}^{-1}$. In summary, in these four cases, we only detect one plausible C IV source at $z=5.74$. Combining the modest SFR of the one detection and the three nondetections, our HST observations strongly support that smaller galaxies $\left(\mathrm{SFR}_{\mathrm{Ly} \alpha} \lesssim 2 M_{\odot} \mathrm{yr}^{-1}\right)$ are main sources of intergalactic $\mathrm{C}$ IV absorbers, and such small galaxies play a major role in the metal enrichment of the intergalactic medium at $z \gtrsim 5$.
\end{abstract}

Key words: galaxies: high-redshift - intergalactic medium

\section{Introduction}

The detection of metal absorption systems at $z>6$ indicates that the intergalactic medium (IGM) has already been enriched by the end of reionization (e.g., Becker et al. 2006, 2012; Keating et al. 2014). A key question is understanding what the sources of early metals are and how they got distributed throughout the IGM in the post-reionization universe (e.g., Songaila 2001; Davé et al. 2006; Ryan-Weber et al. 2006, 2009; D'Odorico et al. 2010, 2013). Theoretical models suggest that galactic superwinds from star-forming galaxies are a main source of the early IGM enrichment. Oppenheimer \& Davé (2006) explored high-redshift IGM absorbers in cosmological simulations, employing various prescriptions for galactic outflows to enrich the IGM at $z=2-6$. Oppenheimer et al. (2009) and Finlator et al. (2015) studied the relation between galaxies and CIV absorbers in simulations and predicted that strong absorbers are the result of outflows from galaxies with stellar masses $M=10^{7-9} M_{\odot}$ and star formation rates (SFRs) of a few $M_{\odot} \mathrm{yr}^{-1}$. The separation between the C IV absorber and the associated galaxy is tens of kiloparsecs $(\approx 5-50 \mathrm{kpc})$ from the quasar line of sight. Note the virial radius of such small galaxies is $\approx 7-8 \mathrm{kpc}$ at $z \approx 5-6$ (e.g., Wechsler et al. 2002).

At $z>5$, observations of IGM-galaxy interactions are difficult. In the field of the strong C IV absorber J1030+0524 at $z=5.7$, Díaz et al. (2011) reached a minimum UV-based star formation of $\sim 5 M_{\odot} \mathrm{yr}^{-1}$ with deep VLT observations. They

\footnotetext{
${ }^{8}$ Hubble Fellow.
}

identified a galaxy associated with the C IV absorber at an impact parameter of $79 \mathrm{kpc}$. Díaz et al. $(2014,2015)$ further present the large-scale overdensity of Ly $\alpha$ emitters (LAEs) at $z \approx 5.7$ associated with these C IV absorption systems. This survey reached a $\mathrm{Ly} \alpha$-based star formation rate $\left(\mathrm{SFR}_{\mathrm{Ly} \alpha}\right)$ of 5 $M_{\odot} \mathrm{yr}^{-1}$, and the closest CIV-galaxy pair had an impact parameter of $300\left(213 h^{-1}\right) \mathrm{kpc}$. Nevertheless, these great efforts have not ruled out the possibility that fainter galaxies with small impact parameters may be the source of C IV absorption. To better test theoretical simulations, we need to probe galaxies down to stellar masses of $M \lesssim 10^{9} M_{\odot}$ and SFRs of $\approx 1-2 M_{\odot} \mathrm{yr}^{-1}$.

Using Hubble Space Telescope (HST)/Advanced Camera for Surveys (ACS) narrowband ramp filters, we carried out deep narrowband imaging of four IGM C IV absorbers at $z=5-6$. We adjusted the ACS ramp filters to the desired wavelength for Ly $\alpha$ detection to search for LAEs in the vicinity of C IV absorbers. The ACS ramp filter has a monochromatic patch of $40^{\prime \prime} \times 20^{\prime \prime}$, corresponding to an area of $250 \mathrm{kpc} \times$ $125 \mathrm{kpc}$ (physical). With the ACS ramp filters, we are able to reach $3 \sigma \mathrm{SFR}_{\mathrm{Ly} \alpha}$ of $=1-2 M_{*} \mathrm{yr}^{-1}$. Typical $\left(M^{*}\right)$ Lyman break galaxies (LBGs) at $z \approx 5-6$ have SFRs of $\sim 7 M_{\odot} \mathrm{yr}^{-1}$. The depth probed in these observations is much lower than the typical LBG and previous ground-based observations. This depth also allows us to test the theoretical models of galactic outflows (e.g., Oppenheimer et al. 2009), which predict that the C IV sources have SFRs of $\lesssim 1 M_{\odot} \mathrm{yr}^{-1}$. In Section 2 , we introduce the $H S T$ observations. In Section 3, we discuss our observational results and the target selection of galaxy 
Table 1

Summary of the C IV-associated Ly $\alpha$ Emitter Candidates

\begin{tabular}{|c|c|c|c|c|c|c|c|c|c|c|c|c|c|}
\hline R.A. & Decl. & $z_{\mathrm{C} \text { IV }}$ & $\log \left[\frac{N_{\mathrm{C} \mathrm{IV}}}{\mathrm{cm}^{2}}\right]$ & $\begin{array}{c}\mathrm{EW}_{\mathrm{C} \text { IV }} \\
(\AA)\end{array}$ & Filter & NB & F775W & F850LP & $\begin{array}{c}L_{\mathrm{Ly} \alpha} \\
\left(10^{42} \mathrm{erg} \mathrm{s}^{-1}\right)\end{array}$ & $\begin{array}{c}\mathrm{SFR}_{\mathrm{Ly} \alpha} \\
\left(M_{\odot} \mathrm{yr}^{-1}\right)\end{array}$ & $\begin{array}{c}\mathrm{SFR}_{\mathrm{UV}} \\
\left(M_{\odot} \mathrm{yr}^{-1}\right)\end{array}$ & $\begin{array}{c}b \\
(\mathrm{kpc})\end{array}$ & $\begin{array}{c}\mathrm{EW}_{\mathrm{Lyc}} \\
(\AA)\end{array}$ \\
\hline \multirow[t]{2}{*}{ 10:30:26.746 } & $+5: 24: 59.76$ & 5.744 & $14.00 \pm 0.04$ & 1.3 & FR853 & $25.90 \pm 0.21$ & $2 \sigma \geqslant 28.0$ & $27.10 \pm 0.13$ & $1.75 \pm 0.44$ & 2.0 & 4.1 & 42 & $44 \AA$ \\
\hline & & 5.517 & $14.02 \pm 0.04$ & 0.6 & FR782 & $2 \sigma \geqslant 26.8$ & $2 \sigma \geqslant 27.9$ & $2 \sigma \geqslant 28.0$ & $3 \sigma \leqslant 1.3$ & $3 \sigma \leqslant 1.2$ & $3 \sigma \leqslant 1.5$ & $\geqslant 125$ & \\
\hline 10:30:27.486 & $+05: 25: 20.15$ & 4.948 & $13.76 \pm 0.11$ & 0.5 & FR716 & $25.24 \pm 0.12$ & $26.21 \pm 0.06$ & $26.04 \pm 0.06$ & $3.27 \pm 0.56$ & 3.0 & 8.7 & 163 & $28 \AA$ \\
\hline 13:06:06.161 & $+03: 56: 32.94$ & 4.866 & $14.80 \pm 0.01$ & 2.6 & FR716 & $25.70 \pm 0.17$ & $27.23 \pm 0.12$ & $27.56 \pm 0.19$ & $2.72 \pm 0.55$ & 2.5 & 2.1 & 205 & $76 \AA$ \\
\hline
\end{tabular}



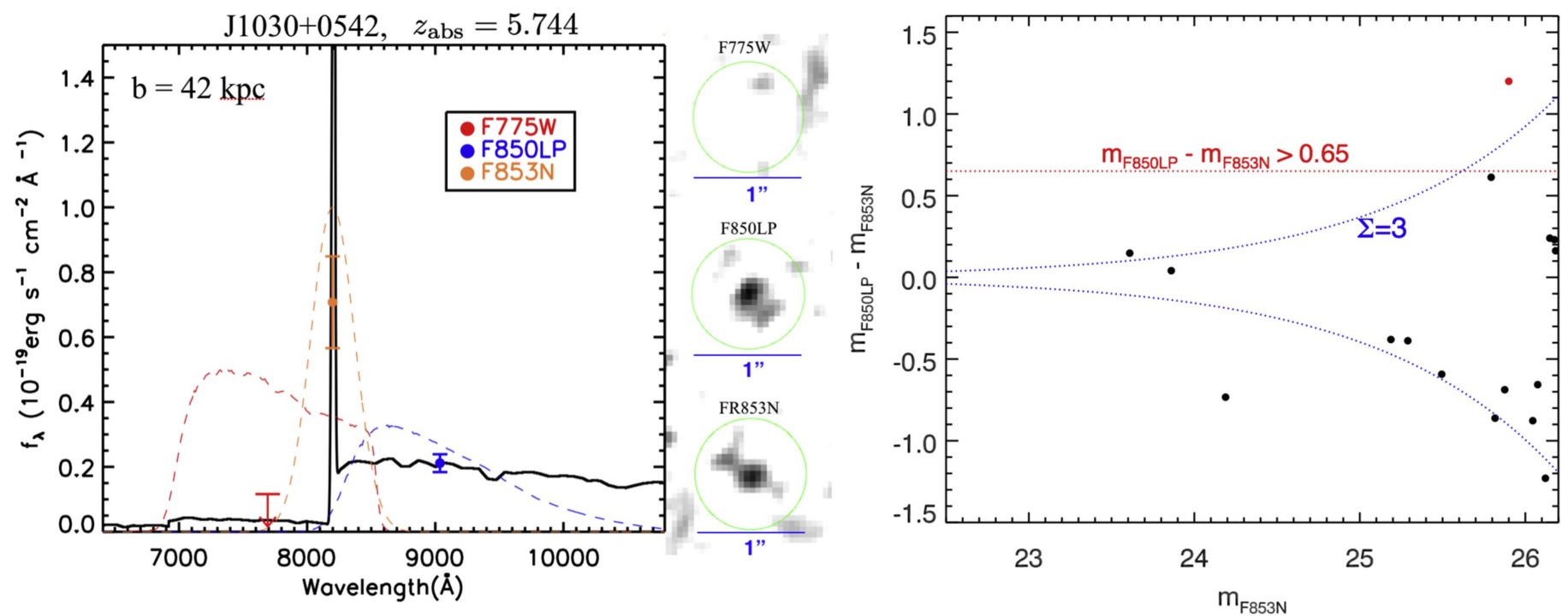

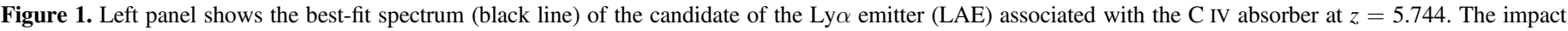

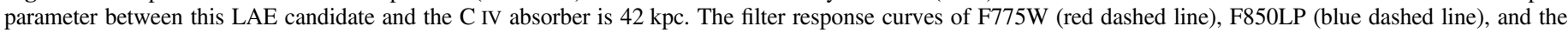

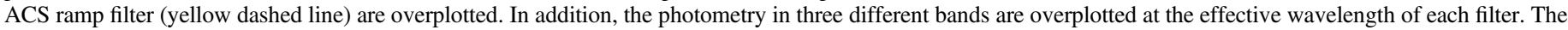

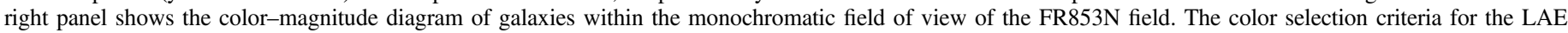

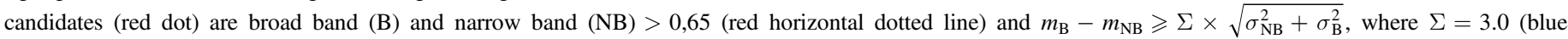
dotted line).

candidates. In Section 4, we discuss the implication of the observations. We convert redshifts to physical distances assuming a $\Lambda$ CDM cosmology with $\Omega_{m}=0.3, \Omega_{\Lambda}=0.7$, and $h=0.70\left(h_{70}\right)$. Throughout this Letter, we use physical distances and $\mathrm{AB}$ magnitude.

\section{Hubble Space Telescope Observations}

\subsection{Sample Selection}

The C IV absorbers at $z=5-6$ were selected from the sample of Simcoe et al. (2011). From this sample, we select four secure, strong C IV absorbers at $z=4.87-5.74$ in two QSO sightlines: SDSS J1030+0524 $\left(z_{\mathrm{QSO}}=6.30\right)$ and SDSS J1306 $+0356\left(z_{\mathrm{QSO}}=6.00\right)$. The $\mathrm{C}$ IV column densities range from 13.8 to $14.8 \mathrm{~cm}^{-2}$. These two QSO fields have broadband (F775W, F850LP) images available from Stiavelli et al. (2005). The sample is summarized in Table 1.

\subsection{Observations}

We devoted 15 orbits to observe four C IV absorber fields in different filters (HST-GO-12860). We used five orbits using the FR853N ramp filter for the $z=5.7$ system, four orbits using F782N for the $z=5.5$ absorber, and three orbits using F716N for each of the two $z=4.9$ systems. For each ramp filter, the central wavelength is tuned to the C IV absorption to search for the Ly $\alpha$ emission associated with $\mathrm{C}$ IV absorption. The ramp filter has a peak efficiency $(70 \%)$ spanning $3000 \mathrm{~km} \mathrm{~s}^{-1}$, much larger than the systematic velocity offset of Ly $\alpha$ to the C IV absorber (450 $\mathrm{km} \mathrm{s}^{-1}$; Steidel et al. 2010). Our HST observations allow us to measure: (1) $\mathrm{SFR}_{\mathrm{Ly} \alpha}$, (2) projected distances between the galaxies and C IV absorbers, and (3) UV-based star formation rates $\left(\mathrm{SFR}_{\mathrm{UV}}\right)$ or their upper limits. The observations probe $\mathrm{SFR}_{\mathrm{Ly} \alpha}=2 M_{\odot} \mathrm{yr}^{-1}$ at the $4 \sigma$ level, corresponding to a Ly $\alpha$ flux of $2.2 \times 10^{42} \mathrm{erg} \mathrm{s}^{-1}$ (Kennicutt 1998). Data reduction is performed with Multidrizzle (Koekemoer et al. 2013). Using a uniform 0 ". 6 diameter aperture, we calculated the depth of our observations in different bands. The narrowband magnitude for point sources reached the $5 \sigma$ level of $m_{\mathrm{F} 716 \mathrm{~N}}=26.1$, $m_{\mathrm{F} 782 \mathrm{~N}}=26.0$, and $m_{\mathrm{F} 853 \mathrm{~N}}=26.1$. The broadband observations reached $m_{\mathrm{F} 850 \mathrm{LP}}=27.8$ and $m_{775 \mathrm{~W}}=27.7$ at the $5 \sigma$ level. Photometry is performed with SExtractor (Bertin \& Arnouts 1996) using the rms map converted from the inverse variance image generated by Multidrizzle (Casertano et al. 2000).

\section{Results}

\subsection{Selection of Ly $\alpha$ Emitter Candidates}

We select LAE candidates with rest-frame equivalent width (EW) $>20 \AA$. The EW of $20 \AA$ corresponds to the color selection criteria of $m_{\text {broadband }}-m_{\text {narrowband }}>0.65$. Also, we require the signal-to-noise ratio of the continuum-subtracted flux to be greater than 3 . The signal-to-noise is calculated using $m_{\mathrm{B}}-m_{\mathrm{NB}} \geqslant \Sigma \times \sqrt{\sigma_{\mathrm{NB}}^{2}+\sigma_{\mathrm{B}}^{2}}$, where $\Sigma=3.0$ and $m_{\mathrm{NB}}, \sigma_{\mathrm{NB}}$, $m_{\mathrm{B}}$, and $\sigma_{\mathrm{B}}$ represent the magnitudes and errors of the narrow band and broad band. The selection criteria are shown in Figures 1-3. Galaxies residing above both red and blue dotted lines are selected. One LAE candidate satisfies the selection criteria in each of the three C IV absorber fields at $z=5.74$, $z=4.95$, and $z=4.87$. We visually checked individual exposures of these candidates to make sure that (a) their photometries are not affected by cosmic rays, and (b) sources do not reside at the detector edges where the photometry is less accurate. We do not detect any LAE candidates associated with the C IV system at $z=5.52$.

In each figure, we use the following galaxy model to fit the photometry of LAE candidates to derive the flux of Ly $\alpha$ emission and the Ly $\alpha$-based and UV-based SFR. We use the Bruzual \& Charlot (2003) models with a Salpeter initial mass function, a 0.2 solar metallicity $(Z=0.004)$, a continuous star formation history, and an age of $30 \mathrm{Myr}$ (e.g., Ono et al. 2010). We use the Ly $\alpha$ optical depth derived by Madau (1995) to account for the IGM absorption. Then, we add the dust extinction following Calzetti et al. (2000) with $R_{V}=$ 

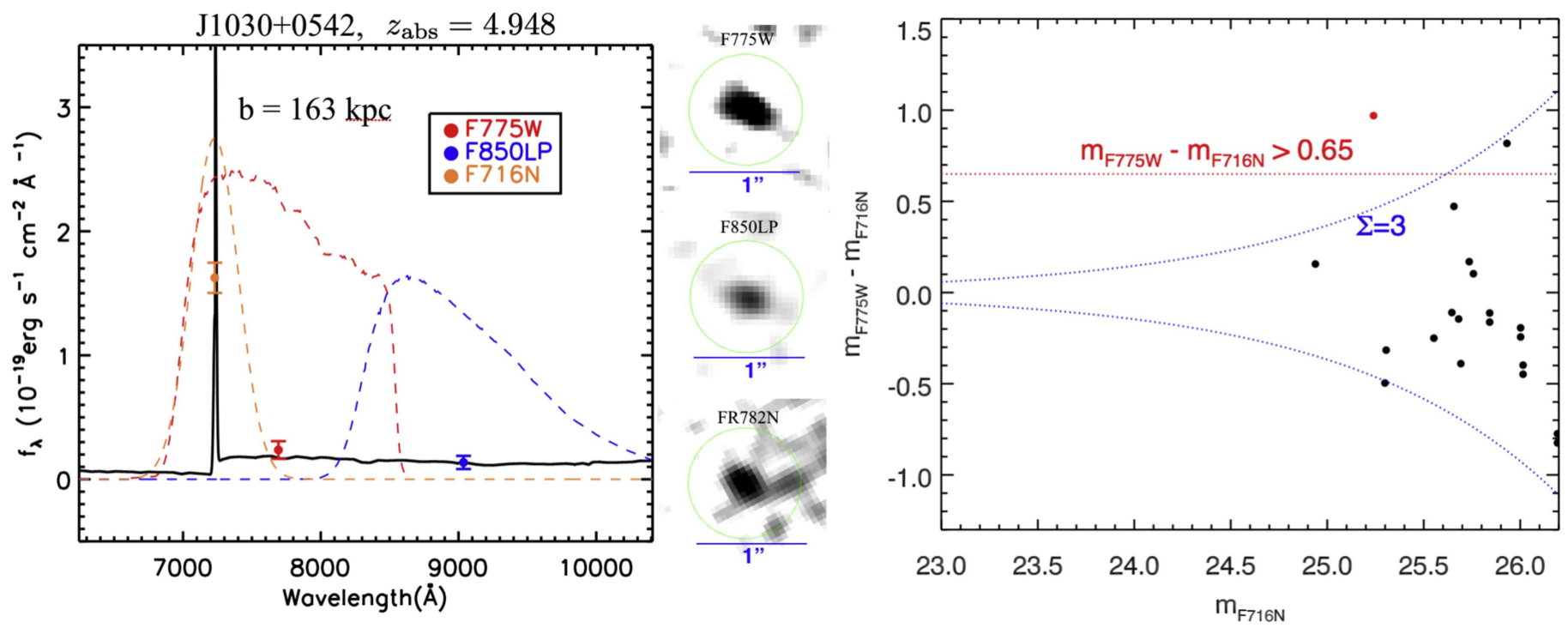

Figure 2. Same format as Figure 1, but presenting the photometry and the selection of LAE candidates in the C IV absorber field at $z=4.948$.

$A(V) / E(B-V)=3.1$ for the $z \approx 5.7$ candidates, and we set $R_{V}$ as a free parameter for two $z \approx 5.0$ candidates. Further, we added the Ly $\alpha$ emission based on our narrowband observations, assuming the FWHM of Ly $\alpha$ is $300 \mathrm{~km} \mathrm{~s}^{-1}$ (e.g., Jiang et al. 2013).

\subsection{Physical Properties of LAE Candidates}

In Table 1, we provide the physical properties of the three LAE candidates. For the C IV absorber at $z_{\text {abs }}=5.744$, we detect a LAE candidate with an impact parameter of $42 \mathrm{kpc}$. This LAE candidate has an expected Ly $\alpha$ luminosity of $L_{\mathrm{Ly} \alpha}=(1.75 \pm 0.44) \times 10^{42} \mathrm{erg} \mathrm{s}^{-1}$, corresponding to the $\mathrm{SFR}_{\mathrm{Ly} \alpha}$ of $2.0 M_{\odot} \mathrm{yr}^{-1}$ (e.g., Kennicutt 1998). The $\mathrm{SFR}_{\mathrm{UV}}$ is estimated to be $4.1 M_{\odot} \mathrm{yr}^{-1}$, using the flux density of the best-fit spectral energy distribution (SED) around $\lambda_{\text {rest }}=1500 \AA$ (Madau et al. 1998). Díaz et al. (2011) found an LAE at $79 \mathrm{kpc}$ away from the CIV absorber and suggested that this LAE could be the powering source. We do not detect any NB excess of this source in our deeper HST observations. This source has $m_{\mathrm{F} 853 \mathrm{~N}}>26.30$, $m_{\mathrm{F} 775 \mathrm{~W}}=26.75 \pm 0.09, \quad m_{\mathrm{F} 850 \mathrm{LP}}=26.23 \pm 0.06, \quad$ which is inconsistent with an LAE at $z \approx 5.7$.

For the C IV absorber at $z_{\mathrm{abs}}=4.948$, the impact parameter of the LAE candidate is $163 \mathrm{kpc}$. The Ly $\alpha$ luminosity is $L_{\mathrm{Ly} \alpha}=(3.27 \pm 0.56) \times 10^{42} \mathrm{erg} \mathrm{s}^{-1}$, corresponding to a $\mathrm{SFR}_{\mathrm{Ly} \alpha}=3.0 M_{\odot} \mathrm{yr}^{-1}$. The $\mathrm{SFR}_{\mathrm{UV}}$ is 8.7 $M_{\odot} \mathrm{yr}^{-1}$. For the $\mathrm{CIV}$ absorber at $z_{\mathrm{abs}}=4.866$, there is an LAE candidate with an impact parameter of $205 \mathrm{kpc}$. The Ly $\alpha$ luminosity is $L_{\mathrm{Ly} \alpha}=(2.72 \pm 0.55) \times 10^{42} \mathrm{erg} \mathrm{s}^{-1}$, corresponding to an $\mathrm{SFR}_{\mathrm{Ly} \alpha}=2.5 \mathrm{M}_{\odot} \mathrm{yr}^{-1}$. The UV-based SFR is estimated to be $2.1 M_{\odot} \mathrm{yr}^{-1}$. If we further assume a stellar mass $\left(M_{*}\right)$-SFR relation at $z=4-5$ (e.g., Sparre et al. 2014), the SFR $_{\mathrm{UV}}$-derived stellar mass of these three LAE candidates have stellar masses of $\sim 10^{9.0-9.5} M_{\odot}$ and halo masses of $\sim 10^{10} M_{\odot}$.

For the C IV absorber at $z_{\text {abs }}=5.517$, we do not detect any LAE associated with this absorber, placing a $3 \sigma$ upper limit on the Ly $\alpha$ luminosity of $1.60 \times 10^{42} \mathrm{erg} \mathrm{s}^{-1}$ and a $3-\sigma$ upper limit on the $\mathrm{SFR}_{\mathrm{Ly} \alpha} \approx 1.5 M_{\odot} \mathrm{yr}^{-1}$.

The main contaminants of high- $z$ LAEs are strong [O II] emitters at lower redshift. For two C IV absorbers at $z \approx 4.9$, we currently have no imaging observations bluer than the Ly $\alpha$ emission. We expect that the contamination rate due to low- $z$
[O II] emission is relatively high. [O II] emitters at $z=0.91$ $(z=0.94)$ with rest-frame $\mathrm{EW}>61.3 \AA$ are contaminants of LAEs at $z=4.948(z=4.866)$ with rest-frame EW $>20 \AA$. From the $[\mathrm{O}$ II] luminosity function at $z \approx 1.0$ (Takahashi et al. 2007; Zhu et al. 2009), strong [O II] emitters that can be detected in our depth $\left(\mathrm{EW}>61.3 \AA L_{[\mathrm{OII}}>10^{41.0} \mathrm{erg} \mathrm{s}^{-1}\right)$ have a number density of $0.009 \mathrm{Mpc}^{-3}$, corresponding to $\approx 0.2$ per field. For LAE candidates at $z=5.7$, a number of studies have been conducted at similar redshifts (e.g., Shimasaku et al. 2006; Ouchi et al. 2008, 2017). Spectroscopic confirmations suggest that the contamination rate is $\lesssim 20 \%$ at our depth. Future spectroscopic follow-ups must be conducted to confirm or rule out the LAE candidates conclusively.

\section{Discussions}

We find three LAE candidates in the field of three strong C IV absorbers at $z=5.744, z=4.948$, and $z=4.866$, respectively. At $z=5.74$, we detect an i-dropout LAE candidate at an impact parameter of 42 physical kpc from the quasar sight line. This is a plausible C IV source that has an $\mathrm{SFR}_{\mathrm{Ly} \alpha}$ of $2 M_{\odot} \mathrm{yr}^{-1}$ and $\mathrm{SFR}_{\mathrm{UV}}=4.1 M_{\odot} \mathrm{yr}^{-1}$. At $z=4.95$ and $z=4.87$, we detect two galaxy candidates with impact parameters of 160 and $200 \mathrm{kpc}$. Here, let us assume galaxies start an outflow at the average redshift when reionization occurs $(z \approx 9$ from Planck observations; Planck Collaboration et al. 2016), then at $z \approx 5.7$, the momentum-driven winds $\left(v \approx 200 \mathrm{~km} \mathrm{~s}^{-1}\right)$ travel about $90 \mathrm{kpc}$ less than the impact parameter we observed. Also, simulations (e.g., Oppenheimer et al. 2009) predict that impact parameters should be $\lesssim 50 \mathrm{kpc}$ to yield strong C IV absorption with $N_{\mathrm{C} \text { IV }} \gtrsim 10^{14} \mathrm{~cm}^{-2}$. We do not detect LAEs associated with the C IV absorber at $z=5.52$. We place $3 \sigma$ upper limits of $\mathrm{SFR}_{\mathrm{Ly} \alpha} \approx 1.5 M_{\odot} \mathrm{yr}^{-1}$ in each of the three nondetection fields.

\subsection{Probing C IV Enrichment Using LAEs}

One potential issue is that not all star-forming galaxies have Ly $\alpha$ emission that can be selected using the LAE selection technique. Schenker et al. (2012) showed that $60 \%$ galaxies with an absolute magnitude of $-20.3<M_{\mathrm{UV}}<-18.7$ have Ly $\alpha$ EW greater than $20 \AA$. Our observations probe galaxies 

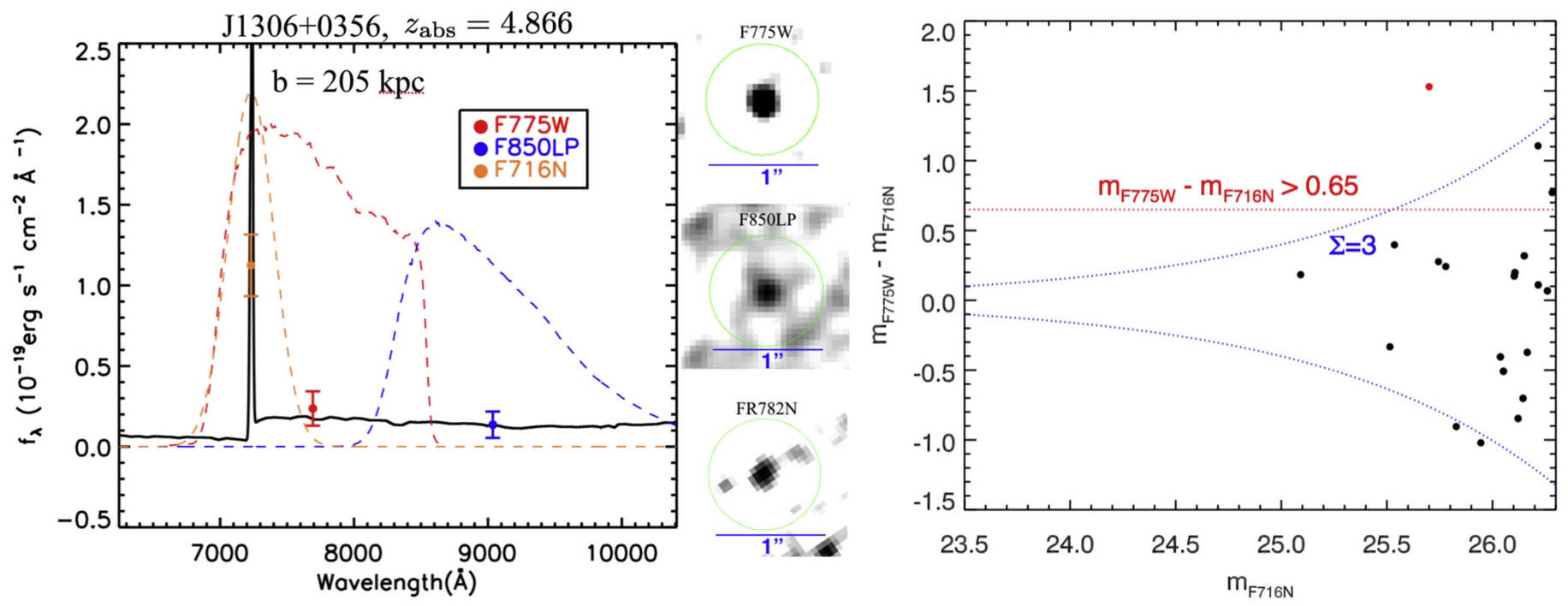

Figure 3. Same format as Figure 1, but showing the photometry and the selection of LAE candidates in the C IV absorber field at $z=4.866$.

down to $M_{\mathrm{UV}} \approx-18.5$ in $3 \sigma$, indicating a high completeness of using the LAE technique to select galaxies in our survey. Another issue is whether LAE candidates we detected are physically associated with the C IV absorbers. Observationally, we can evaluate this issue by calculating the number of galaxies expected in random fields: if $\ll 1$ galaxies are expected within an impact parameter in random fields, then galaxies detected within these impact parameters are more likely to be sources of C IV systems (e.g., Díaz et al. 2014). This is because C IV absorbers are expected to be clustered with the physically associated galaxies. The LAE candidate we detected at $z=5.74 \pm 0.07$ is the first source reported at $z>5$ to be projected $\approx 40 \mathrm{kpc}$ away from a C IV absorber. From the luminosity function at $z=5.7$ (Ouchi et al. 2008; Konno et al. 2017), the $L_{\mathrm{Ly} \alpha}^{*}=6.8 \times 10^{42} \mathrm{erg} \mathrm{s}^{-1}$ and $\phi^{*}=$ $7.7 \times 10^{-4} \mathrm{Mpc}^{-3}$. Our limiting Ly $\alpha$ luminosity is 0.26 $\times L_{\mathrm{Ly} \alpha}^{*}$. The width of the FR853N ramp filter corresponds to a redshift interval of $\Delta z=0.14$. The expected number of finding an LAE in random fields with $L_{\text {Lya }} \geqslant 0.26 \times L_{\text {Ly } \alpha}^{*}$ and at impact parameters of $\leqslant 42 \mathrm{kpc}$ is 0.01 . The number of faint galaxies with $L=0.01-0.26 \times L_{\mathrm{Ly} \alpha}^{*}$ (below our detection limit) and an impact parameter within $42 \mathrm{kpc}$ is 0.2 . Thus, there is a relatively small probability of finding such an LAE in random fields. If this LAE candidate is confirmed by future deep spectroscopy, then one can conclude that this galaxy is highly likely to be the source of the C IV absorber at $z=5.744$.

For two CIV absorbers at $z \approx 5$, the expected number of LAEs with $L_{\mathrm{Ly} \alpha} \geqslant 0.26 \times L_{\mathrm{Ly} \alpha}^{*}$ and an impact parameter of $\approx 200 \mathrm{kpc}$ is 0.5 . The number of faint LAEs $\left(L_{\mathrm{Ly} \alpha}=\right.$ $\left.0.01-0.26 \times L_{\mathrm{Ly} \alpha}^{*}\right)$ is expected to be $\approx 10$ in random fields. As a result, it is unlikely that the LAE candidates we detected at $z \approx 5.0$ are sources of the C IV absorbers, and thus we treat these two sightlines as nondetections.

\subsection{Constraining IGM Enrichment Models}

From our one detection and three nondetections, we conclude that strong C IV systems at $z \approx 5-6$ do not generally lie close to galaxies identified as LAEs with SFR $\gtrsim 2 M_{\odot} \mathrm{yr}^{-1}$. This is surprising because strongly star-forming galaxies are expected to be driving the strongest outflows at these epochs, enriching the early IGM. Models generically predict early, widespread enrichment of metals (e.g., Madau et al. 2001; Oppenheimer \& Davé 2008), such that $z \gtrsim 5$ represents the first epoch of IGM enrichment. Given this, the most straightforward interpretation of our result is that early enrichment is dominated, at least in a volumetric sense, by small galaxies with SFR $\lesssim 2 M_{\odot} \mathrm{yr}^{-1}$.

There are two important caveats to this result. First, our observations cannot select LBGs without strong Ly $\alpha$ emission. However, as noted in the previous section, LBGs and LAEs are often coincident at these epochs (60\% of LBGs at $z=5-6$ are LAEs; e.g., Schenker et al. 2012). If the $40 \%$ that are not LAEs are randomly associated with LBGs, then the probability of all four C IV systems being undetected is $0.4^{4}(<3 \%)$. Second, our selection disfavors the detection of massive dust-obscured galaxies. Such galaxies are metal-rich and have high SFRs, so potentially we could be missing these sources. However, typical high-SFR objects at these epochs should not be heavily dust-obscured (e.g., Ono et al. 2010; Finkelstein et al. 2012). Future submillimeter observations would be required to rule out this explanation conclusively.

Setting aside the caveats, our conclusion represents an important constraint on models of early metal production and dissemination. Such models began by postprocessing metal distributions onto cosmological simulations (e.g., Davé et al. 1998; Aguirre et al. 2001), but the introduction of outflows into simulations by Springel \& Hernquist (2003) enabled selfconsistent IGM enrichment. Oppenheimer \& Davé (2006) showed that a momentum-driven outflow model in which small galaxies have higher mass loading (i.e., outflow rate relative to SFR) yielded better agreement with C IV enrichment at $z \sim 2-4$, as compared to models where the mass loading is independent of galaxy mass. Oppenheimer et al. (2009) extended these results to $z \sim 6$ and showed that the high mass loading factors in small galaxies resulted in early enrichment in accord with C IV data, despite a fairly small metal volume filling factor of a couple percent. They further predicted that strong absorbers $\left(N_{\text {C IV }}=10^{13.8-14.8} \mathrm{~cm}^{-2}\right)$ arise from outflows from galaxies with stellar masses $\sim 10^{7-9} M_{\odot}$ and impact parameters from a few kiloparsecs up to $50 \mathrm{kpc}$. These predictions are consistent with our observational results. 
Using cosmological simulations, Finlator et al. (2013) introduced on-the-fly radiative transfer into cosmological simulations and examined early metal enrichment in conjunction with an inhomogeneous ionizing background to determine the impact of local UVB fluctuations on the ionization state. They argued that the mass scale of halos hosting O I absorbers was $10-100 \times$ smaller than typical LBGs at $z \sim 6$, which is consistent with our results if OI and C IV absorption arises around similar systems. In contrast, Keating et al. (2016) examined early IGM enrichment in the Sherwood and Illustris simulations and found that galaxies associated with strong $(\mathrm{EW}>1 \AA$ ) $\mathrm{C}$ IV absorbers have halo masses of $M>10^{11} M_{\odot}$ and impact parameters of $10-30 \mathrm{kpc}$. These results seem to be less in agreement with our results at face value, although a more careful comparison is warranted. This is interesting because Illustris utilizes a mass loading factor that increases to lower masses even more rapidly than in momentum-driven wind models. Hence, other factors such as local density and ionization may be playing a key role. It could be that the Keating et al. (2016) and Nelson et al. (2015) models are predicting strong $\mathrm{C}$ IV from dust-obscured galaxies that would be missed by our LBG/LAE survey.

To summarize, we present the detection of one plausible C IV source at $z=5.7$ with a projected distance of $42 \mathrm{kpc}$ and an $\mathrm{SFR}_{\mathrm{Ly} \alpha}=1.75 \pm 0.44 M_{\odot} \mathrm{yr}^{-1}$. Also, we found two LAE candidates in the field of two C IV absorbers at $z=4.95$ and $z=4.87$ with projected distances of 163 and $205 \mathrm{kpc}$, respectively. But the impact parameters are much larger than that predicted by simulations, making them unlikely to be C IV sources. We do not detect C IV-associated LAEs at $z=5.52$. From our observations, no C IV-associated LAEs have impact parameters $\lesssim 40 \mathrm{kpc}$ with $\mathrm{SFR}_{\mathrm{Ly} \alpha} \geqslant 1.5 M_{\odot} \mathrm{yr}^{-1}$ (3- $\sigma$ limit). Particularly, for the strongest absorbers with EW $>2 \AA$, we do not detect LAEs with $3 \sigma \quad \mathrm{SFR}_{\mathrm{Ly} \alpha} \geqslant 1.4 \quad M_{\odot} \mathrm{yr}^{-1}$. Our observations provide strong constraints on models of early enrichment in simulations. Future facilities, including the James Webb Space Telescope, will probe the IGM-galaxy relation down to a much fainter galaxy population where some models predict that the C IV arises.

We thanks the referee for valuable comments. Z.C. thanks Marcel Neeleman for his useful comments. Z.C. and X.F. thank the support from the US NSF grant AST 11-07682. Support for part of this work was also provided by NASA through the Hubble Fellowship grant HST-HF2-51370 awarded by the Space Telescope Science Institute, which is operated by the Association of Universities for Research in Astronomy, Inc., for NASA, under contract NAS 5-26555.

\section{ORCID iDs}

Zheng Cai ib https://orcid.org/0000-0001-8467-6478 Xiaohui Fan (1) https://orcid.org/0000-0003-3310-0131 Romeel Dave (D) https://orcid.org/0000-0003-2842-9434
Ben Oppenheimer (Di) https://orcid.org/0000-0002-3391-2116

\section{References}

Aguirre, A., Hernquist, L., Schaye, J., et al. 2001, ApJ, 561, 521

Becker, G. D., Sargent, W. L. W., Rauch, M., \& Carswell, R. F. 2012, ApJ, 744, 91

Becker, G. D., Sargent, W. L. W., Rauch, M., \& Simcoe, R. A. 2006, ApJ, 640, 69

Bertin, E., \& Arnouts, S. 1996, A\&AS, 117, 393

Bruzual, G., \& Charlot, S. 2003, MNRAS, 344, 1000

Calzetti, D., Armus, L., Bohlin, R. C., et al. 2000, ApJ, 533, 682

Casertano, S., de Mello, D., Dickinson, M., et al. 2000, AJ, 120, 2747

Davé, R., Finlator, K., \& Oppenheimer, B. D. 2006, MNRAS, 370, 273

Davé, R., Hellsten, U., Hernquist, L., Katz, N., \& Weinberg, D. H. 1998, ApJ, 509,661

Díaz, C. G., Koyama, Y., Ryan-Weber, E. V., et al. 2014, MNRAS, 442, 946

Díaz, C. G., Ryan-Weber, E. V., Cooke, J., Koyama, Y., \& Ouchi, M. 2015, MNRAS, 448, 1240

Díaz, C. G., Ryan-Weber, E. V., Cooke, J., Pettini, M., \& Madau, P. 2011, MNRAS, 418, 820

D'Odorico, V., Calura, F., Cristiani, S., \& Viel, M. 2010, MNRAS, 401, 2715

D’Odorico, V., Cupani, G., Cristiani, S., et al. 2013, MNRAS, 435, 1198

Finkelstein, S. L., Papovich, C., Salmon, B., et al. 2012, ApJ, 756, 164

Finlator, K., Muñoz, J. A., Oppenheimer, B. D., et al. 2013, MNRAS, 436, 1818

Finlator, K., Thompson, R., Huang, S., et al. 2015, MNRAS, 447, 2526

Jiang, L., Egami, E., Mechtley, M., et al. 2013, ApJ, 772, 99

Keating, L. C., Haehnelt, M. G., Becker, G. D., \& Bolton, J. S. 2014, MNRAS, 438, 1820

Keating, L. C., Puchwein, E., Haehnelt, M. G., Bird, S., \& Bolton, J. S. 2016, MNRAS, 461, 606

Kennicutt, R. C., Jr. 1998, ARA\&A, 36, 189

Koekemoer, A. M., Ellis, R. S., McLure, R. J., et al. 2013, ApJS, 209, 3

Konno, A., Ouchi, M., Shibuya, T., et al. 2017, PASJ, in press (arXiv:1705. 01222)

Madau, P. 1995, ApJ, 441, 18

Madau, P., Ferrara, A., \& Rees, M. J. 2001, ApJ, 555, 92

Madau, P., Pozzetti, L., \& Dickinson, M. 1998, ApJ, 498, 106

Nelson, D., Pillepich, A., Genel, S., et al. 2015, A\&C, 13, 12

Ono, Y., Ouchi, M., Shimasaku, K., et al. 2010, ApJ, 724, 1524

Oppenheimer, B. D., \& Davé, R. 2006, MNRAS, 373, 1265

Oppenheimer, B. D., \& Davé, R. 2008, MNRAS, 387, 577

Oppenheimer, B. D., Davé, R., \& Finlator, K. 2009, MNRAS, 396, 729

Ouchi, M., Harikane, Y., Shibuya, T., et al. 2017, PASJ, in press (arXiv:1704. 07455)

Ouchi, M., Shimasaku, K., Akiyama, M., et al. 2008, ApJS, 176, 301

Planck Collaboration, Adam, R., Aghanim, N., et al. 2016, A\&A, 596, A108

Ryan-Weber, E. V., Pettini, M., \& Madau, P. 2006, MNRAS, 371, L78

Ryan-Weber, E. V., Pettini, M., Madau, P., \& Zych, B. J. 2009, MNRAS, 395, 1476

Schenker, M. A., Stark, D. P., Ellis, R. S., et al. 2012, ApJ, 744, 179

Shimasaku, K., Kashikawa, N., Doi, M., et al. 2006, PASJ, 58, 313

Simcoe, R. A., Cooksey, K. L., Matejek, M., et al. 2011, ApJ, 743, 21

Songaila, A. 2001, ApJL, 561, L153

Sparre, M., Hartoog, O. E., Krühler, T., et al. 2014, ApJ, 785, 150

Springel, V., \& Hernquist, L. 2003, MNRAS, 339, 289

Steidel, C. C., Erb, D. K., Shapley, A. E., et al. 2010, ApJ, 717, 289

Stiavelli, M., Djorgovski, S. G., Pavlovsky, C., et al. 2005, ApJL, 622, L1

Takahashi, M. I., Shioya, Y., Taniguchi, Y., et al. 2007, ApJS, 172, 456

Wechsler, J. S., Primack, J. R., Kravtsov, A. V., \& Dekel, A. 2002, ApJ, 568,52

Zhu, G., Moustakas, J., \& Blanton, M. R. 2009, ApJ, 701, 86 